\title{
Perfil actual del paciente en hemodialisis hospitalaria. Análisis de sus necesidades
}

\author{
$M^{a}$ Dolores Contreras Abad \\ $M^{a}$ Flora Rivero Arellano \\ $M^{a}$ Jesús Jurado Torres \\ Rodolfo Crespo Montero
}

\author{
Servicio de Nefrología \\ Hospital Universitario Reina Sofía \\ Córdoba
}

\section{RESUMEN}

Con el propósito de reflejar nuestra experiencia en el cuidado del paciente en hemodiálisis hospitalaria, se realizó este estudio con los objetivos de detectar el grado de dependencia de los pacientes dializados en nuestra unidad y su repercusión en la necesidad de cuidados de enfermería.

Se realizó un estudio descriptivo transversal a los pacientes en hemodiálisis hospitalaria, entre los meses de noviembre y diciembre de 2002. Fueron estudiados 38 pacientes, 19 mujeres y 19 hombres, que llevaban de tiempo medio 84 meses en diálisis (2-317); y con un rango de edad entre 21-78 años. 13 pacientes eran diabéticos (34\%). Para un mejor análisis según la edad, se agruparon según edades: 30-65 años (19 pacientes), 65-70 años (7 pacientes), 70-80 años (12 pacientes). De las 14 necesidades básicas (V. Henderson), elegimos cinco, aquellas que más pueden influir en la necesidad de cuidados de enfermería en diálisis hospitalaria (Comer y beber, Eliminación, Moverse, Vestirse, Higiene). La dependencia se analizó globalmente, en cada una de las necesidades estudiadas y separada por grupos de edad.

En la necesidad de Comer y beber: $42 \%$ eran dependientes. Eliminar: 36\% eran dependientes. Mo- verse: 73,6\% eran dependientes. Vestirse: 68,3\% eran dependientes. Higiene: 55,1\% eran dependientes. Cuando se agruparon por edades, en la necesidad de Comer y beber, en el grupo I el 36\% eran dependientes, en el grupo II el 28,4\% y en el grupo III, el 58,2\%. Respecto a Eliminación, en el grupo I el 26\% eran dependientes, en el grupo II el $14,2 \%$ y en el grupo III el $58,2 \%$. En cuanto a Moverse, en el grupo I el 62\% eran dependientes, en el grupo II el 71,3\% y en el grupo III el 91,6\%. En cuanto a Vestirse, el 57,8\% eran dependientes en el grupo I, en el grupo II el $57 \%$ y en el grupo III el $91,6 \%$. Finalmente respecto a la Higiene, en el grupo I, el $42 \%$ eran dependientes, en el grupo II el $28,5 \%$ y en el grupo III el $74,9 \%$ eran dependientes. 11 pacientes (29\%) eran trasladados directamente de la camilla de la ambulancia a la cama de diálisis, mediante una grúa sanitaria. Otros 12 pacientes (32\%) utilizaban sillas de ruedas para el traslado a la unidad.

En conclusión, los pacientes de edad igual o superior a 60 años con patología crónica, presentan mayor grado de dependencia, por lo que van a necesitar cuidados individualizados y adaptados a esta realidad. Las dependencias que presentan los pacientes que tenemos actualmente en nuestra unidad, confirman un aumento cuantitativo y cualitativo de cuidados, ante las necesidades que estos plantean.

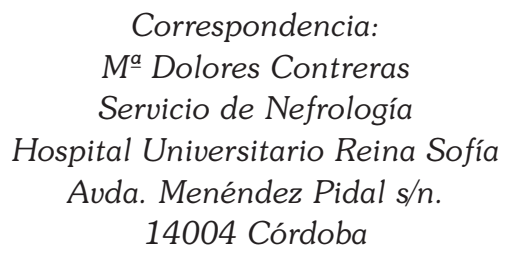

\section{PALABRAS CLAVE: HEMODIÁLISIS HOSPITALARIA \\ PACIENTE ANCIANO \\ NECESIDADES BÁSICAS}




\section{CURRENT PROFILE OF PATIENT UNDER HE- MODIALYSIS. AN ANALYSIS OF THEIR NEEDS.}

\section{SUMMARY}

This study was performed with the aim of detecting the degree of dependence of patients under hemodialysis and its influence on the necessity of nursing care.

We performed a transversal descriptive study in patients under hemodialysis between November and December 2002. We studied 38 patients, 19 women and 19 men who were under hemodialysis for 84 months (2-317) with a range of age between 21 and 78 years. 13 patients (34\%) were diabetic. Patients were grouped according to their age, 30 - 65 years ( 19 patients), 65 - 70 years ( 7 patients), $70-80$ years (12 patients). From the 14 basic needs (V Henderson) we chose those 5 that can have the greatest influence on nursing care (food and drink, elimination, movement, dressing, hygiene). Dependence was analysed globally.

For food and drink $42 \%$ were dependent. Elimination, 36\% were dependent. Moving 73.6\% were dependent. Dressing 68.3\% were dependent. Hygiene $55.1 \%$ were dependent. When patients were grouped by age, for food and drink, in group one, $36 \%$ were dependent, in group 2, 28\% and in group 3, 58\%. For elimination in group 1, 26\% were dependent, in group 2, 14\%, and in group 3, $58 \%$. For moving, in group 1, 62\% were dependent, in group 2, $71 \%$ and in group 3, 92\%. For dressing, in group 1, 58\% were dependent, in group 2, 57\%, and in group 3, 92\%. Finally, for hygiene, in group 1, $42 \%$ were dependent, in group 2, 28\%, and in group 3, 75\%.

In conclusion, patients of 60 years or older, with chronic pathology present a higher degree of dependence. Therefore, they need individual care adapted to this reality. Areas of dependence presented by patients in our unit, confirm a quantitative and qualitative increase in caring procedures.

KEY WORDS:
HOSPITAL HEMODIALYSIS

ELDERLY PATIENT

BASIC NEEDS

\section{INTRODUCCIÓN}

En los últimos años estamos asistiendo a cambios sustanciales en la población de pacientes con insuficiencia renal terminal (IRT) en tratamiento de hemodiálisis (HD) periódicas $(1,2)$. El número de pacientes que acuden a las unidades de diálisis con alguna discapacidad, secundaria a su patología de base, es cada vez mayor $(3,4)$. Este hecho, es un reflejo exacto de lo que está ocurriendo en la población general, con una supervivencia y expectativas de vida, impensables hace unas décadas. Gracias a los avances científicos y médicos, hoy se sobrevive a enfermedades graves y a problemas que antes eran mortales, pero por contra, esta supervivencia va asociada a diferentes grados de incapacidad en muchos casos. Aparece entonces la dependencia en sus diferentes formas y la necesidad de una mayor atención por parte de los profesionales de salud y de la sociedad en general. Este hecho general es fácilmente reproducible en las unidades de HD donde la población atendida es cada vez más anciana (5-7).

La aparición de más dependencias relacionadas con el aumento de la edad y otras patologías asociadas de los pacientes atendidos en nuestra unidad, ha hecho que enfermería nos hayamos tenido que adaptar a esta nueva situación, ofreciendo unos cuidados dirigidos no solo al tratamiento sustitutivo, sino a satisfacer una serie de necesidades, que han aumentado considerablemente el tiempo de enfermería dedicado a cada paciente $(8,9)$.

Tradicionalmente la asignación de enfermera/paciente en las unidades de diálisis se ha realizado de forma cuantitativa, 4-5 pacientes/enfermera (10). Esta ratio, que pudiera ser válido en algunos centros periféricos, no lo es en modo alguno para pacientes en HD hospitalaria, donde la medida de las necesidades de cuidados debe llevar a una asignación cuanti-cualitativa basada en el nuevo perfil de los pacientes en diálisis.

Con estos antecedentes nos propusimos reflejar nuestra experiencia en el cuidado del paciente en HD hospitalaria, con los objetivos de detectar el grado de dependencia de los pacientes dializados en nuestra unidad y su repercusión en la necesidad de cuidados de enfermería.

\section{PACIENTES Y MÉTODOS}

Se llevó a cabo un estudio descriptivo en el que se realizó un corte transversal de la población de pacientes en HD hospitalaria de nuestra unidad, en los meses de noviembre y diciembre de 2002.

Fueron estudiados 38 pacientes, 19 mujeres y 19 hombres, que llevaban 84 meses de tiempo medio en diálisis 
(rango entre 2 y 317 meses); y con un rango de edad entre 21-78 años. Trece pacientes eran diabéticos (34\%). Para un mejor análisis según la edad, se agruparon según la clasificación de Aragó (1986) en las siguientes fases y edades:

- 30-65 años. Edad adulta: 19 pacientes.

- 65-70 años. Tercera edad: 7 pacientes.

- 70-80 años. Ancianidad: 12 pacientes.

- > 90 años. Cuarta edad: 0 pacientes.

Marco conceptual: Nos basamos en el análisis de las necesidades de los pacientes según modelo de Virginia Henderson donde la persona presenta 14 necesidades fundamentales de orden bio-físico-social, entendiendo como necesidad lo que resulta indispensable a la persona para mantenerse en vida y asegurar su bienestar. De estas 14 necesidades, hemos elegido cinco, precisamente aquellas que más pueden influir en la necesidad de cuidados de enfermería durante HD hospitalaria: $\mathrm{Co}^{-}$ mer y beber, Eliminación, Moverse, Vestirse, Higiene. Estas cinco necesidades se analizaron en función de que los pacientes fueran Independientes o Dependientes, para satisfacerlas o realizarlas por si mismos. Teniendo en cuenta que en el adulto se considera INDEPENDENCIA la consecución de un nivel aceptable de satisfacción de sus necesidades mediante acciones apropiadas que el sujeto realiza él mismo sin ayuda de ninguna otra persona; y DEPENDENCIA la incapacidad del sujeto para adaptar comportamientos o realizar por si mismo, sin ayuda de otra persona, las acciones susceptibles de permitirle un nivel aceptable de satisfacción de sus necesidades.

El grado de dependencia se analizó de forma global para todos los pacientes en cada una de las necesidades estudiadas y separada por grupos de edad.

Se estudió además el tipo de acceso vascular, la necesidad de grúa sanitaria, carritos u otro tipo de ayuda para el acomodo de los pacientes en la cama, las curas de úlceras por presión o vasculares y otros tratamientos no habituales administrados durante la sesión de HD.

La recogida de datos se obtuvo de los registros y la entrevista con el paciente o familiar, y en el conocimiento empírico de los pacientes. Los datos se representan como distribución de frecuencias.

\section{RESULTADOS}

Del total, 11 pacientes (29\%) eran trasladados directamente de la camilla de la ambulancia a la cama de diálisis, mediante grúa sanitaria con la ayuda de al menos dos sanitarios (celador, auxiliar enfermería, enfermera). Otros 12 pacientes (32\%) utilizaban sillas de ruedas pa- ra el traslado a la unidad, necesitando también ayuda auxiliar para su acomodo en la cama. Diez pacientes (26\%) entraban en las salas de diálisis acompañados hasta la cama, siendo ayudados a introducirse en la cama; 5 pacientes (13\%) no necesitaban ayuda, ni para entrar en la sala ni acostarse en la cama.

A cinco pacientes se les realizaban curas de úlceras por decúbito o úlceras vasculares y a dos se les realizaban curas de la colostomía.

En cuanto a los datos obtenidos en la valoración de las necesidades de la totalidad de los pacientes están representados en la Tabla I.

Tabla I.- Porcentaje de pacientes dependientes (o semidependientes) e independientes, según las necesidades estudiadas para el total de la población estudiada.

\begin{tabular}{|l|c|c|}
\hline NECESIDADES & \% dependientes & \% independientes \\
\hline Comer y beber & 42 & 58 \\
\hline Eliminar & 37 & 63 \\
\hline Moverse & 72 & 28 \\
\hline Vestirse & 68 & 32 \\
\hline Higiene & 55 & 45 \\
\hline
\end{tabular}

Cuando se agruparon por edades, en los tres subgrupos descritos anteriormente, encontramos para cada uno de ellos los siguientes resultados:

La figura 1 muestra la necesidad de Comer y beber representando a los pacientes agrupados por edad según clasificación de Aragó, observando que en el grupo III el $\%$ de pacientes dependientes es mayor que el de pacientes independientes. La figura 2 representa a los tres grupos de edad para la necesidad de Eliminar, observándose también que en el grupo III el \% de dependientes es mayor que el \% de pacientes independientes. En la figura 3

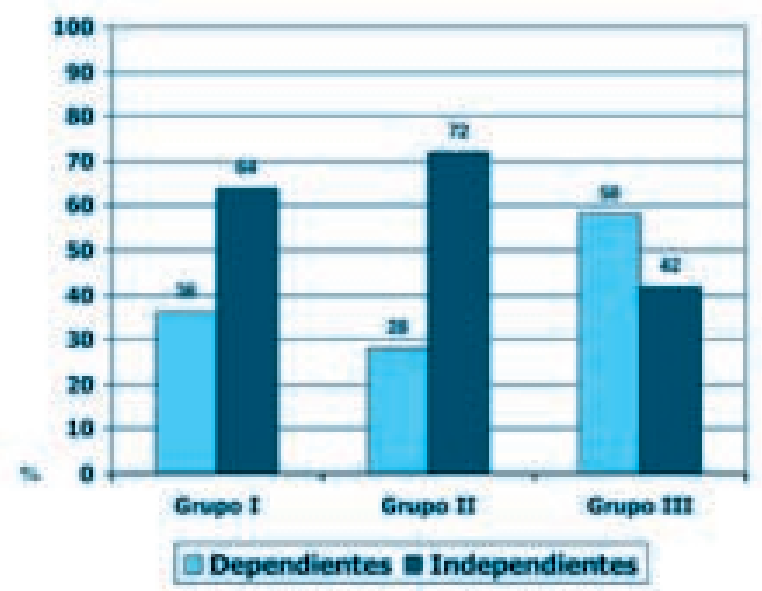

Figura 1: Porcentaje de pacientes dependientes e independientes para satisfacer la necesidad de COMER Y BEBER. 


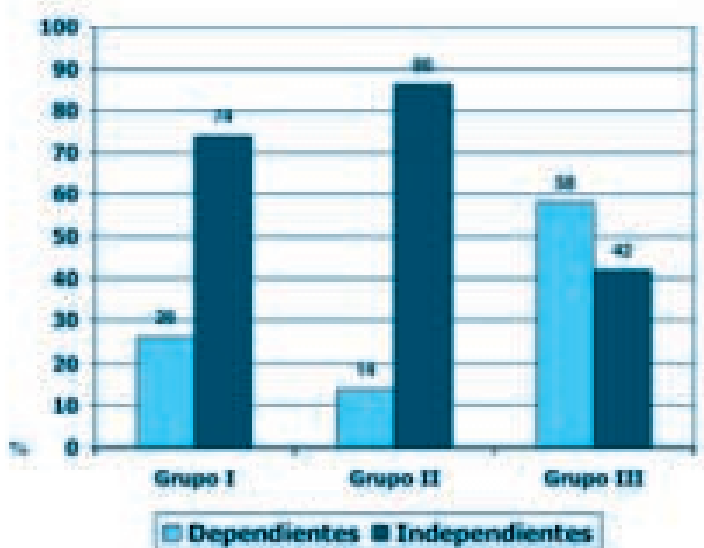

Figura 2: Porcentaje de pacientes dependientes e independientes para satisfacer la necesidad de ELIMINAR.

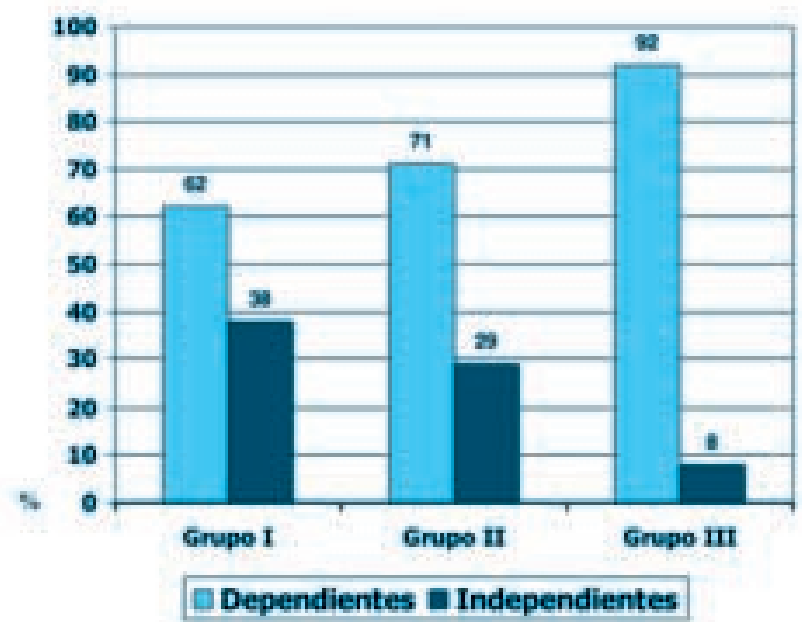

Figura 3: Porcentaje de pacientes dependientes e independientes para satisfacer la necesidad de MOVERSE.

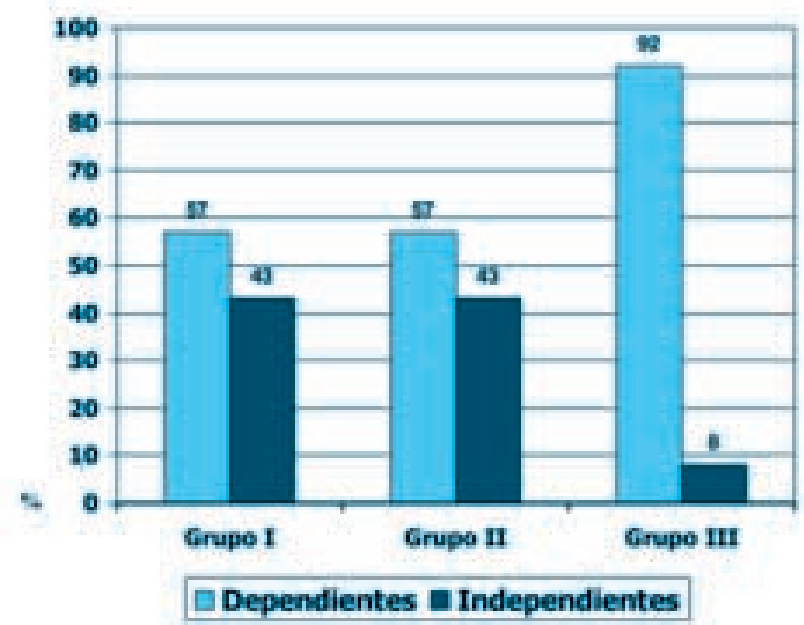

Figura 4: Porcentaje de pacientes dependientes e independientes para satisfacer la necesidad de VESTIRSE.

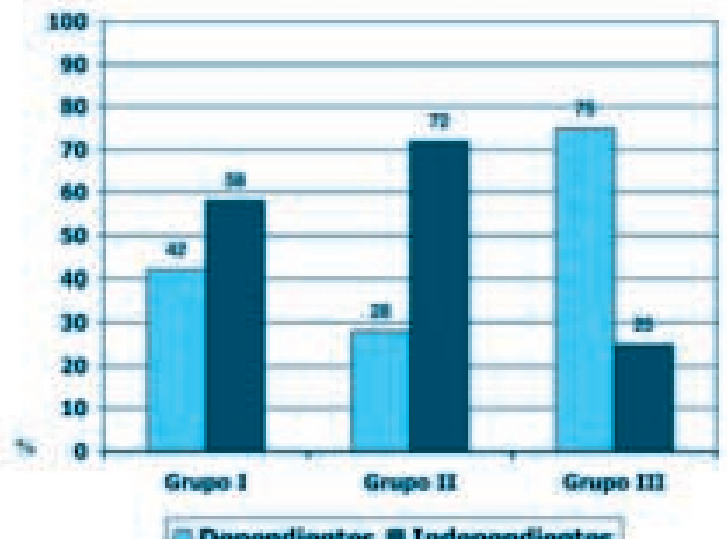

Figura 2: Porcentaje de pacientes dependientes e independientes para satisfacer la necesidad de realizarse la HIGIENE.

se representan los tres grupos para la necesidad de Moverse, observándose que en los tres grupos de edad, el \% de pacientes dependientes es superior al de independientes y es especialmente notoria la diferencia en el grupo III (91,6 frente 8,3\%). La figura 4, representa asimismo la necesidad de vestirse, observándose también que en los tres grupos de edad, el porcentaje de pacientes dependientes es superior al de independientes, siendo más acusada la diferencia en el grupo III (91,6 frente 8,3\%). En cuanto a la última necesidad analizada, la Higiene, está representada en la figura 5, observándose también, que en el grupo III el \% de dependientes es mayor que el \% de pacientes independientes.

Respecto al acceso vascular 19 pacientes eran portadores de una fístula arteriovenosa interna y 19 eran portadores de un catéter tunelizado o "permanente". En el último mes se tuvieron que aplicar 18 protocolos de desobstrucción de estos catéteres.

Un paciente recibía nutrición parenteral durante la HD, cuya preparación se realiza en la Unidad.

\section{DISCUSIÓN}

El grado de dependencia encontrado en nuestros pacientes, provocado por disfunciones físicas tales como, retinopatía diabética con ceguera total o parcial, amputaciones de uno o dos miembros por la misma causa, prótesis de cadera que limitan la movilidad en pacientes ancianos así como poliartritis con inmovilidad total o parcial, se viene traduciendo en un aumento en la demanda de cuidados de enfermería por parte de estos pacientes, como también reflejan otros estudios $(3,8)$.

Es significativo que la mayor dependencia apareció tanto en la totalidad de pacientes como por grupos de edad 
en las necesidades de moverse y vestirse lo que implica para enfermería un aumento en las cargas de trabajo relacionadas con el manejo físico del paciente: pesarles, ayudarles a vestirse, acostarles, movilizarles en la cama, ayudarles a comer, lo que unido a que los catéteres presentan con frecuencia problemas de permeabilidad y/o flujo sanguíneo, aumenta los tiempos de conexión y desconexión a la HD (9).

Por otra parte, la incapacidad para satisfacer la necesidad de estar limpio, cuidado y proteger los tegumentos que afectó al 55\% del total de pacientes y al 74,9\% (figura 5) en el grupo de mayor edad, junto a la inmovilidad total o parcial que un porcentaje bastante elevado sufría, favoreció la aparición de escaras y ulceras por presión en cinco de nuestros pacientes. Este hecho ha supuesto para enfermería una actualización en el tratamiento y prevención de este problema para poder realizar de forma eficaz y adecuada las curas que esta alteración necesita.

La siguiente necesidad alterada, en cuanto a porcentaje total, fue la de comer y beber. El 42\% tenían dificultad para alimentarse o hidratarse adecuadamente, padeciendo un paciente una severa desnutrición tratada con nutrición parenteral. Esto ha generado un aumento de las cargas de trabajo de las auxiliares de enfermería, al tener que preparar, administrar o facilitar la ingesta de alimento o líquidos durante la sesión de diálisis, a casi la mitad de nuestros pacientes.

En cuanto a la necesidad de eliminar, el 36,7\% eran dependientes o semidependientes traduciéndose esto en incontinencia fecal, lo que les obligaba a hacer uso de pañales, teniéndose que realizar con frecuencia, higiene íntima de algunos pacientes durante las sesiones de HD. Así como las curas y cuidados de las colostomías de dos pacientes.

En cuanto al estudio de las dependencias por grupos de edad, señalar que las dependencias en las necesidades estudiadas presentaron una tendencia clara, a mayor edad mayor dependencia, como puede apreciarse en las figuras 1-5. Esta tendencia es generalizable tal como destacan otros autores (11-13) y generando un profundo debate en la comunidad científica $(2,14,15,16)$. En nuestro caso, además, la mitad (49,9\%) de los pacientes pertenecían al grupo de edad superior a 65 años.

Otro aspecto cualitativo a destacar, es el cuidado y manejo del acceso vascular, que se ve afectado en gran medida por el perfil actual del paciente en HD. El grupo estudiado eran pacientes mayores con múltiples patologías en los que había sido difícil conseguir una buena fístula para HD, y otros que llevaban en diálisis mucho tiempo y que han agotado todas las posibilidades de fístulas autólogas o injertos, por lo que el numero de pacientes portadores de catéteres tunelizados ha aumentado de forma considerable. Aunque datos recientes han comunicado que aproximadamente el $10 \%$ de los pacientes en HD de nuestro país se dializan a través de un catéter tunelizado o "permanente" (17), en nuestra unidad el 50\% de los pacientes eran portadores de fístulas y el 50\% catéteres tunelizados o permanentes, lógico por otra parte, pues en la unidad hospitalaria, se concentran los pacientes con más problemas. Esto ha supuesto un aumento del tiempo de conexión de los pacientes a la HD (9), por los problemas que plantean las fístulas "complejas" con dificultades de punción o los catéteres que no funciona adecuadamente, siendo frecuente tener que realizar protocolos fibrinolíticos en los catéteres (en el mes estudiado se realizaron 8 protocolos de uroquinasa).

Por último, es necesario reflexionar sobre el desgaste emocional que el cuidado de estos pacientes, ocasiona en enfermería. Acompañar tan de cerca y continuado en el tiempo, el sufrimiento sin esperanza de algunos de estos pacientes, es muy duro, apareciendo en nosotros sentimientos contradictorios de pena y alivio cuando alguno fallece (18).

Como principales conclusiones, hemos comprobado que las dependencias que presentan los pacientes dializados en la unidad hospitalaria, confirman que se ha producido un incremento cuantitativo y cualitativo de cuidados para dar respuesta a las necesidades que estos presentan.

Paralelo al incremento de los cuidados, apareció un aumento del tiempo que enfermería dedica a la aplicación de los mismos, aunque esta variable no se ha medido en este estudio.

Los pacientes de edad igual o superior a 60 años con patología crónica, presentan mayor grado de dependencia, por lo que los cuidados deben plantearse de forma individualizada en el paciente anciano y adaptados a su situación.

Hay que reevaluar el ratio enfermera/paciente, comúnmente aceptado, pues el paciente actual en HD hospitalaria, presenta unas dependencias que derivan en mayor necesidad de dedicación de enfermería, lo que sin duda debe abrir nuevas líneas de investigación.

\section{BIBLIOGRAFÍA}

1. Alonso R. Cambios en el perfil clínico de los pacientes en hemodiálisis. En: Nuevo perfil del paciente renal; un reto para los profesionales. VII Semanario Español de la EDTNA/ERCA. Valencia; 11-12 Abril, 1997: 31-40.

2. Ortega F, Gómez E, Baltar J Rebollo P. Controversias en nefrología. Diálisis en el anciano. Nefrología; 2001; XXI (4): 332-336. 
3. Fernández A, Valdor M, Pérez MaI, Sáenz de Buruaga A y Díez M. Cuidando juntos al enfermo en programa de hemodiálisis. Libro de Comunicaciones XXIII Congreso de la SEDEN. Sevilla; 1998:9094.

4. García Pérez R. Pacientes geriátricos en hemodiálisis. Diálisis del anciano. Revista de la Sociedad Española de Enfermería Nefrológica. 2001; 15: 64-73.

5. Amenábar JJ, García F, Robles NR, Caracho R, Pinilla, Gentil MA y cols.: Informe de diálisis y trasplante de la Sociedad Española de Nefrología y Registros Autonómicos, año 2000; Nefrología 2002; XXII (4) 310-317.

6. Comité de Registro de la S.E.N, Comunidades y registros Autonómicos: Informe de Diálisis y Trasplante de la Sociedad Española de Nefrología y Registros Autonómicos correspondiente al año 2001. http://www.senefro.org (consultado el 20/12/2003).

7. E.R.A.-E.D.T.A. Registry: Annual Report 2001. http://www.era-edta-reg.org/annep.jsp/ (consultado el 20/12/2003).

8. Estany N, Pérez M ${ }^{a}$ A, Serra A, Sorolla C, Alegri M, Fuentes C. Análisis del grado de dependencia relacionado con la edad biológica de los pacientes afectos de IRCT. ¿Es preciso una valoración geriátrica? Libro de Comunicaciones XXI Congreso de la SEDEN. Salamanca; 1996: 378-385.

9. Fuentes C, Pérez García MaA A, Colón L, y cols. Análisis de las cargas de trabajo en pacientes en hemodiálisis. Estudio multicéntrico la provincia de Girona. Libro de Comunicaciones XXV Congreso de la SEDEN. Oviedo; 2000:123-127.

10. Balhorn J. Clasificación de pacientes utilizada como herramienta en la valoración de los ratios personal/pacientes. Edtna-Erca Journal 1998; XXIV (1):15-19.
11. Alvarez Ude F. Factores asociados al estado de salud percibido de los pacientes en hemodiálisis crónica. Revista de la Sociedad Española de Enfermería Nefrológica. 2001; 14: 64-68.

12. Miguel I, López T, Díaz-Cardiel G, Moreno M, Martín S. Estado de salud y calidad en pacientes en diálisis. Libro de Comunicaciones XXV Congreso de la SEDEN. Oviedo; 2000:153-158.

13. Muñoz C, Martín A, Millán M. Tolerancia y calidad de vida en ancianos hemodiálisis. Revista de la Sociedad Española de Enfermería Nefrológica. 2001; 14: 92-93.

14. Ashwanden C. El cuidado del paciente renal de edad avanzada. EDTNA-ERCA Journal, 2000; XXVI (3):47-50.

15. Bernaert P. Cuidado del paciente geriátrico con insuficiencia renal crónica. EDTNA-ERCA Journal, 2001; XXVII (2):158-161.

16. Rebollo P, Ortega F, Badía X, y cols. Salud percibida en pacientes mayores de 65 años en tratamiento sustitutivo renal. Nefrología 1999; XIX supl 1: 73-83.

17. Rodríguez JA, López Pedret J, Piera L. El acceso vascular en España: análisis de su distribución, morbilidad y sistemas de monitorización. Nefrología 2001; 1 21:45-54.

18. Nieto B, Alvarez R, Castaño A, López A, Martínez E, Ureña A. Perfil de los pacientes con insuficiencia renal terminal que fallecen en la unidad de hospitalización. Libro de Comunicaciones XXIV Congreso de la SEDEN. Valencia; 1999:210-212. 